\title{
Effet du stress salin sur l'accumulation de la proline et des sucres solubles dans les feuilles de trois porte-greffes d'agrumes au Maroc
}

\author{
Mustapha Ait Haddou Mouloud $^{a \star}$, Aouatif Bousrhal ${ }^{b}$, Hamid Benyahia ${ }^{a}$, Abdelkader Benazzoouz $^{a}$
}

a Laboratoire d'Agrophysiologie, Programme national de recherche sur les agrumes, Inra,

El Menzeh, BP1055,

Kenitra, Maroc

(aithaddou_com@yahoo.com)

b Faculté des Sciences, Université Ibn Tofail, Kenitra, Maroc

\section{Effect of saline stress on proline and soluble sugar accumulation in three citrus rootstock leaves in Morocco.}

Abstract - Introduction. Soil salinity and irrigation water, worsened by the rarity of rainfalls, are a permanent threat to the cultivation of citrus trees in Morocco. The choice of a rootstock able to tolerate this abiotic stress would be an effective means of attenuating this problem. The experimentation presented aimed to evaluate three citrus rootstocks recently introduced to Morocco, regarding saline stress. Materials and methods. Seedlings of citrange Carrizo and two hybrids, H1 and H2, resulting from the crossing (Poncirus trifoliata $\times$ Citrus sunki Hort. ex Tan.) were evaluated for their tolerance to salinity. The 18-month-old seedlings were irrigated by a nutritive solution containing $(25,35$, and 70$) \mathrm{mM}$ of $\mathrm{NaCl}$ for one or two months, whereas the control seedlings received the nutritive solution without $\mathrm{NaCl}$. After treatment, the proline and soluble sugar contents were measured in the leaves of the treated rootstocks. Results. The accumulation of proline was independent of the presence of $\mathrm{NaCl}$ in the irrigation solution but it varied according to the rootstock genotype. On the other hand, $\mathrm{NaCl}$ strongly stimulated the accumulation of soluble sugars in the three rootstocks and this accumulation was very significant in the citrus rootstock H1. Discussion and conclusions. These results agree partly with some of the work already published. Under our experimental conditions, the effect of the $\mathrm{NaCl}$ concentrations used could be compensated by the storage of soluble sugars in the leaves, in particular in those of the rootstock H1. As this showed less foliar toxicity than the two other rootstocks, the soluble sugar storage in the citrus leaves could indicate a tolerance to salinity.

Morocco / Citrus / rootstocks / salt tolerance / leaf extracts / sugars / proline

\section{Effet du stress salin sur l'accumulation de la proline et des sucres solubles dans les feuilles de trois porte-greffes d'agrumes.}

Résumé - Introduction. La salinité du sol et de l'eau d'irrigation, aggravée par la rareté des précipitations, est une menace permanente pour la culture des agrumes au Maroc. Le choix d'un porte-greffe tolérant à ce stress abiotique serait un moyen efficace pour pallier ce problème. L'expérimentation présentée a permis d'évaluer, vis-à-vis du stress salin, trois portegreffes récemment introduits au Maroc. Matériel et méthodes. Des plants de citrange Carrizo et de deux hybrides, H1 et H2, issus du croisement Poncirus trifoliata x Citrus sunki Hort. ex Tan. ont été évalués pour leur tolérance à la salinité. Les plants âgés de 18 mois ont été irrigués par une solution nutritive additionnée de $(25,35$, et 70$) \mathrm{mM}$ de $\mathrm{NaCl}$ durant un ou deux mois alors que les plants témoins recevaient la solution nutritive sans $\mathrm{NaCl}$. Après traitement, les teneurs en proline et en sucres solubles ont été mesurées dans les feuilles des porte-greffes traités. Résultats. L'accumulation de proline a été indépendante de la présence de $\mathrm{NaCl}$ dans la solution d'irrigation mais elle a varié en fonction du porte-greffe. En revanche, $\mathrm{NaCl}$ a fortement stimulé l'accumulation des sucres solubles chez les trois porte-greffes et cette accumulation a été très importante chez le porte-greffe H1. Discussion et conclusions. Ces résultats concordent en partie avec certains des travaux déjà publiés. Dans nos conditions expérimentales, l'effet des concentrations salines utilisées a pu être compensé par l'accumulation de sucres solubles dans les feuilles, notamment dans celles du porte-greffe H1. Celui-ci ayant montré moins de toxicité foliaire que les deux autres porte-greffes, l'accumulation de sucres solubles dans la feuille d'agrumes pourrait être un indice de tolérance à la salinité.

Maroc / Citrus / porte greffe / tolérance au sel / extrait de feuille / sucres / proline 


\section{Introduction}

Dans certaines régions, la salinité du sol est une menace qui pèse de manière permanente sur l'agriculture. Elle est stimulée par la sécheresse et aggravée par un mauvais drainage dans le sol.

Au Maroc, les agrumes sont généralement cultivés dans des zones où les quantités de pluie reçues sont insuffisantes. Par conséquent, les agriculteurs ont souvent recours à l'irrigation pour améliorer la production. Cependant, les rendements en vergers d'agrumes peuvent être gravement compromis par l'accumulation des sels dans le sol $[1,2]$, problème qui touche actuellement les régions semi-arides du pays où ces arbres sont largement cultivés [3]. Par ailleurs, cette contrainte abiotique prédispose les porte-greffes à une gommose parasitaire due aux Phytophthora spp. [4].

Les agrumes sont particulièrement sensibles aux sels [1]. Leur tolérance à la salinité varie d'une espèce à l'autre et dépend principalement du porte-greffe utilisé. L'effet le plus néfaste des sols salins est dû à la présence d'ions chlorures plus ou moins bien assimilés par le porte-greffe [1]. La sélection de porte-greffes résistants à la salinité est généralement faite sur la base de son aptitude à exclure le chlore et le sodium $[5,6]$. Dans ce cas, le dosage de la concentration du chlore dans les différentes parties de la plante (racine, tige et feuille) permet de fournir des informations sur le degré de résistance des porte-greffes au passage du chlore dans la plante. Un porte-greffe qui, en conditions de salinité, pourrait supporter de fortes accumulations de tels ions serait alors tolérant. Ainsi, il nous a déjà été donné de constater qu'un porte-greffe donné pouvait manifester relativement moins de symptômes nécrotiques qu'un autre porte-greffe ayant assimilé presque la même quantité de chlore [7].

Cependant, la tolérance d'une plante au stress salin pourrait aussi être attribuée à sa capacité d'accumuler des substances non toxiques capables de protéger ses organites cellulaires contre les effets néfastes du chlore et du sodium particulièrement $[2,8]$. D'autres travaux $[9,10]$ ont démontré que la salinité stimule la protéolyse en augmentant l'accumulation d'acides aminés libres, de la proline notamment. Par ailleurs, selon Fernandez-Ballester et al. [8], une forte teneur en carbohydrates totaux et en sucres solubles chez le bigaradier serait liée à une augmentation de la teneur de ses feuilles en ions chlorures.

Par suite, l'objectif de notre étude a été de vérifier le rôle joué par certaines substances biochimiques telles que la proline et les sucres totaux dans la régularisation de l'activité biochimique de trois porte-greffes d'agrumes, récemment introduits au Maroc et placés en conditions de stress salin.

\section{Matériel et méthodes}

Le bigaradier, porte-greffe communément utilisé au Maroc dans les vergers d'agrumes en vertu de son adaptation aux conditions pédo-climatiques des régions agrumicoles de ce pays, est très sensible à la tristeza, maladie virale présente en Espagne et menaçant les vergers marocains. Récemment, plusieurs porte-greffes tolérant à cette maladie ont été introduits de la station de Recherche agronomique de San Giuliano de Corse (France). Notre étude a donc cherché à évaluer trois de ces nouveaux portegreffes vis-à-vis du stress salin : le citrange Carrizo et deux hybrides 'ICVN 0110211' (H1) et 'ICVN 0110208' (H2) issus d'un croisement entre Poncirus trifoliata (L.) Raf. $\times$ Citrus sunki. Hort. ex Tan.

Au total, 72 plants homogènes âgés de 17 mois, soit 24 plants pour chaque portegreffe, ont été utilisés. Ils ont été transférés dans des pots de $8 \mathrm{~L}$ contenant un substrat stérilisé constitué de sable, tourbe et fumier en parties égales. Les porte-greffes ont été placés sous un tunnel en plastique et irrigués quatre fois par semaine par une solution de Hoagland et Arnon [11] diluée de moitié et dépourvue de chlorure de sodium $(\mathrm{NaCl})$ durant 1 mois avant application d'un stress salin [12].

Le $\mathrm{NaCl}$, qui est le sel le plus répandu dans le sol et dans l'eau d'irrigation des principales régions productrices d'agrumes 
du Maroc [3], a été ensuite ajouté à la solution d'irrigation. Trois doses ont été utilisées : $(25,35$ et 70$) \mathrm{mM}$. Une irrigation constituée de la seule solution nutritive a servi de traitement témoin. Afin d'éviter de provoquer un choc osmotique dans le matériel végétal, les concentrations en $\mathrm{NaCl}$ ont été augmentées progressivement durant 1 semaine dans la solution d'irrigation appliquée [12, 13].

Après (30 et 60) j d'irrigation à l'eau saline, des feuilles matures des porte-greffes ont été prélevées puis analysées quant à leur teneur en proline et en sucres solubles. Le dosage de la proline a été fait selon la méthode de Troll et Lindsley [14] et le dosage des sucres selon la méthode à l'anthrone.

Le dispositif expérimental a été un splitplot avec six répétitions ; le facteur principal étudié a été le porte-greffe. En cas de différences significatives, les moyennes ont été comparées par le test de Duncan au seuil de $5 \%$.

\section{Résultats}

\subsection{Effet de la salinité sur la teneur des feuilles en proline}

L'accumulation de la proline dans les feuilles des porte-greffes s'est révélée être indépendante de la concentration du sel dans la solution d'irrigation, mais cette teneur a varié d'un porte-greffe à l'autre dès $30 \mathrm{j}$ de traitement à l'eau saline (tableau I). Nous n'avons pas mis en évidence d'interaction entre l'effet génotype et l'effet dose de $\mathrm{NaCl}$.

Après $30 \mathrm{j}$ de traitement avec les différentes doses de $\mathrm{NaCl}$, les moyennes des teneurs en proline dans les feuilles des trois porte-greffes ont permis de regrouper les porte-greffes citrange Carrizo et hybride H1 dans un seul groupe homogène avec, respectivement, (139 et 109) $\mu \mathrm{mol} \cdot \mathrm{g}^{-1} \mathrm{de}$ matière sèche (ms), alors que l'hybride $\mathrm{H} 2$ ne contenait que $47 \mu \mathrm{mol} \cdot \mathrm{g}^{-1} \mathrm{~ms}$ (tableau II). Des résultats semblables ont été observés après $60 \mathrm{j}$ de traitement.

\subsection{Effet de la salinité sur la teneur des feuilles en sucres solubles}

Dès $30 \mathrm{j}$ après le début des irrigations avec du $\mathrm{NaCl}$, la teneur en sucres solubles dans les feuilles a été significativement différente dans les porte-greffes testés mais non selon les doses de $\mathrm{NaCl}$ utilisées. Ces doses n'ont induit un effet qu'après $60 \mathrm{j}$ d'irrigation avec le sel (tableau III). Comme dans le cas de la teneur en proline, nous n'avons pas observé d'interaction entre l'effet génotype et l'effet dose de $\mathrm{NaCl}$.

En considérant l'ensemble des portegreffes après ces $60 \mathrm{j}$ de traitement, l'effet imputable au chlore a pu être attribué à la dose de $70 \mathrm{mM}$, alors que les doses à $(25$ et 35) $\mathrm{mM}$ donnaient un effet comparable à l'irrigation témoin sans addition de sel

\section{Tableau I.}

Résultat d'une analyse de variance effectuée sur la teneur en proline des feuilles de trois porte-greffes d'agrumes irrigués avec de l'eau enrichie avec différentes doses de (0 à 70$) \mathrm{mM}$ de $\mathrm{NaCl}$ pendant (30 ou 60) j de traitement.

\begin{tabular}{lcc} 
Source de variation & \multicolumn{2}{c}{ F observée } \\
\cline { 2 - 3 } & Après 30 j de traitement & Après 60 j de traitement \\
Porte-greffe & 6,96 * & $10,01^{*}$ \\
Dose de $\mathrm{NaCl}$ & $0,16 \mathrm{~ns}$ & $0,87 \mathrm{~ns}$ \\
Porte-greffe $\times$ dose & $0,99 \mathrm{~ns}$ & $1,87 \mathrm{~ns}$ \\
& & \\
* Significatif ; ns : non significatif. &
\end{tabular}

\section{Tableau II.}

Teneur en proline ( $\mu \mathrm{mol} \cdot \mathrm{g}^{-1}$ de matière sèche) des feuilles de trois porte-greffes d'agrumes irrigués avec de l'eau enrichie avec différentes doses de $(0$ à 70$) \mathrm{mM}$ de $\mathrm{NaCl}$ soit pendant $30 \mathrm{j}$, soit pendant $60 \mathrm{j}$.

\begin{tabular}{lcr}
\hline Porte-greffe & 30 jours & 60 jours \\
Citrange Carrizo & $139 \mathrm{a}$ & $101 \mathrm{a}$ \\
Hybride H1 & $109 \mathrm{a}$ & $100 \mathrm{a}$ \\
Hybride H2 & $47 \mathrm{~b}$ & $44 \mathrm{~b}$
\end{tabular}

Les moyennes de la même colonne affectées par la même lettre ne diffèrent pas significativement au seuil de $5 \%$ (test de Duncan). 


\section{Tableau III.}

Résultat d'une analyse de variance effectuée sur la teneur en sucres solubles des feuilles de trois porte-greffes d'agrumes irrigués avec de l'eau enrichie avec différentes doses de (0 à 70) $\mathrm{mM}$ de $\mathrm{NaCl}$ pendant (30 ou 60) j de traitement.

\begin{tabular}{lcc} 
Source de variation & \multicolumn{2}{c}{ F observée } \\
\cline { 2 - 3 } & Après 30 j de traitement $\quad$ Après 60 j de traitement \\
& $15,37^{*}$ & 4,36 * \\
Porte-greffe & $0,05 \mathrm{~ns}$ & 0,83 * \\
Dose de $\mathrm{NaCl}$ & $0,22 \mathrm{~ns}$ & $0,90 \mathrm{~ns}$ \\
Porte-greffe $\times$ dose & & \\
& & \\
* Significatif ; ns : non significatif. &
\end{tabular}

Figure 1.

Teneur en sucres solubles dans les feuilles de portegreffes d'agrumes après $60 \mathrm{j}$ d'irrigation avec différentes doses de chlorure de sodium ajoutées à l'eau apportée. Pour chaque traitement, les barres munies des mêmes lettres ne diffèrent pas significativement au seuil de $5 \%$ (test de Duncan).

\section{Figure 2.}

Teneur en sucres solubles dans les feuilles de trois porte-greffes d'agrumes après 60 j d'irrigation avec une solution soit dépourvue de chlore, soit enrichie avec $70 \mathrm{mM}$ de $\mathrm{NaCl}$.
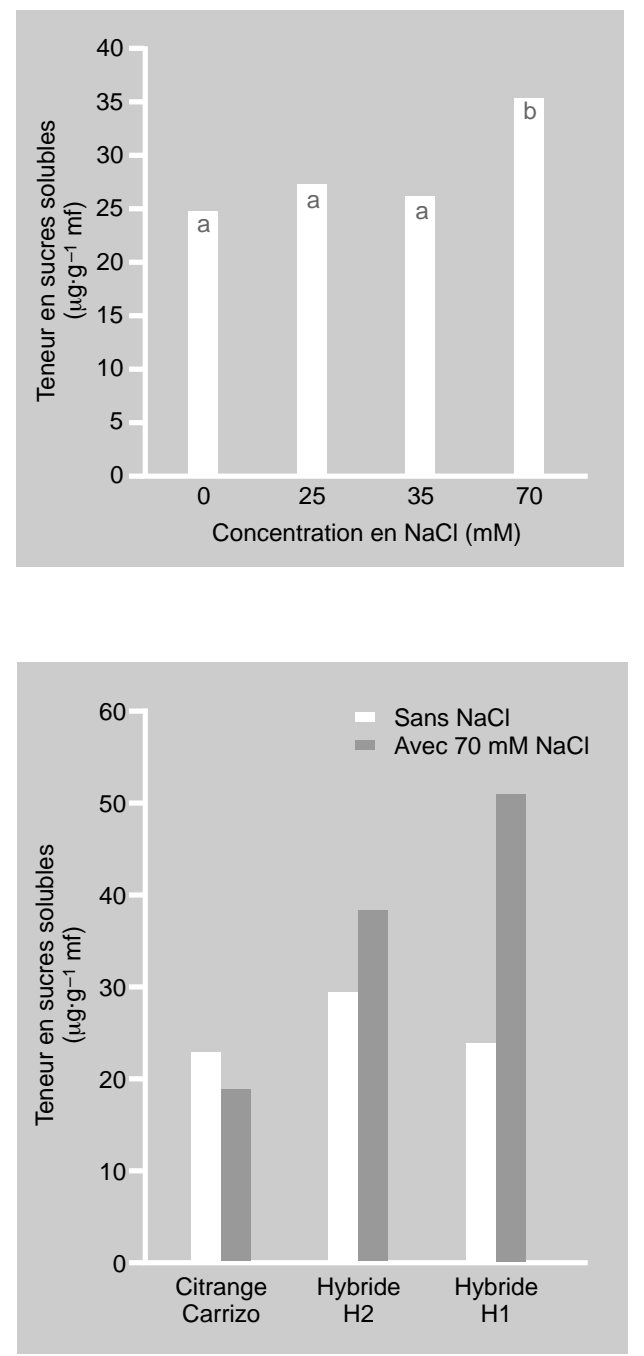

(figure 1). Par ailleurs, l'accroissement du taux de sucres solubles dans les plants traités avec $70 \mathrm{mM}$ de $\mathrm{NaCl}$ a été particulièrement important chez l'hybride $\mathrm{H} 1$ puisqu'il a atteint $50,36 \mu \mathrm{g} \cdot \mathrm{g}^{-1}$ de matière fraîche (mf) dans les feuilles, contre $22,96 \mu \mathrm{g} \cdot \mathrm{g}^{-1}$ mf mesurés chez les témoins non traités (figure 2).

Comme pour la teneur en proline, après 30 j d'irrigation avec $\mathrm{NaCl}$, les teneurs en sucres solubles des feuilles des trois portegreffes ont permis de différencier deux groupes statistiquement différents, mais le regroupement n'a pas été le même : groupe formé par le citrange Carrizo et l'hybride H2 d'une part, groupe constitué par l'hybride H1 $\left(39,81 \mu \mathrm{g} \cdot \mathrm{g}^{-1} \mathrm{mf}\right.$ ) d'autre part (tableau IV).

\section{Discussion et conclusion}

Le stress salin se répercute généralement sur la physiologie des agrumes par un effet osmotique et un déséquilibre nutritionnel [1]. Le maintien des processus physiologiques sous de telles situations est conditionné par l'activation de diverses stratégies notamment l'exclusion des ions toxiques et/ou l'accumulation de substances organiques qui permettent d'éviter le problème de la déshydratation [15].

Dans notre étude, le taux de proline évalué après analyse foliaire n'a pu être relié à la concentation saline de l'eau d'irrigation. Ces résultats vont dans le même sens que ceux obtenus par Fernandez-Ballester et al. [8] selon lesquels une irrigation saline augmenterait légèrement mais non significativement le taux de cet acide aminé dans les feuilles. D'autres auteurs [16] ont rapporté qu'une accumulation de proline serait une caractéristique du porte-greffe. Cependant, selon Hubac et Gerruer [17], la proline ne serait pas la conséquence d'un stress, mais plutôt métabolisée comme facteur de tolérance. Hasson et Poljakof-Mayber [18], quant à eux, auraient mis en évidence une accumulation d'acides aminés dans les feuilles sous l'effet de la salinité et, dans la plupart des cas, il s'agirait de la proline dont le taux d'accumulation augmenterait avec la concentration saline. 
Dans notre étude, l'absence de l'effet du traitement salin sur la teneur en proline pourrait être liée aux concentrations salines utilisées, son accumulation étant alors conditionnée par de plus fortes concentrations de sel que celles utilisées ; selon Townsend, il faudrait en effet de (100 à 150) $\mathrm{mM}$ de $\mathrm{NaCl}$ pour induire une telle accumulation [19]. En outre, il a été démontré que, en conditions de salinité, l'augmentation de la proline dans les feuilles des agrumes serait plutôt un indice de sensibilité que de tolérance.

Contrairement à la proline, le taux en sucres solubles a été fortement stimulé par une irrigation à l'eau saline à $70 \mathrm{mM}$ de $\mathrm{NaCl}$, notamment chez l'hybride $\mathrm{H} 1$. Au cours de notre expérimentation, nous avons constaté que la teneur en eau des tissus foliaires des trois porte-greffes, et plus particulièrement de l'hybride H1, n'était pas affectée par la présence du sel dans la solution d'irrigation (données non fournies). Ce porte-greffe hybride $\mathrm{H} 1$, bien qu'ayant accumulé des quantités de chlore comparables à celles enregistrées pour le portegreffe $\mathrm{H} 2$, a montré moins de symptômes de toxicité foliaire [7]. S'il est admis que les sucres solubles participent à l'ajustement osmotique de la plante placée en conditions de salinité $[2,8]$, nous pouvons avancer que ces solutés auraient pu alors jouer un tel rôle chez l'hybride H1 en atténuant l'effet toxique du chlore. En s'appuyant sur une telle supposition, l'accumulation des sucre solubles dans la feuille d'agrumes pourrait être un indice de tolérance à la salinité

\section{Remerciements}

Les auteurs remercient la station de Recherches agronomiques Inra-Cirad de San Giuliano (Corse, France) pour sa contribution à la sélection de porte-greffes adaptés à la culture des agrumes au Maroc.

\section{Références}

[1] Maas E.V., Salinity and citriculture, Tree Physiol. 12 (2) (1993) 195-216.

\section{Tableau IV.}

Teneur en sucres solubles ( $\mu \mathrm{g} \cdot \mathrm{g}^{-1}$ de matière fraîche) des feuilles de trois porte-greffes d'agrumes irrigués avec de l'eau enrichie avec différentes doses de (0 à 70) $\mathrm{mM}$ de $\mathrm{NaCl}$ pendant $60 \mathrm{j}$.

\begin{tabular}{lr}
\hline Porte-greffe & 1 mois de traite \\
\hline Citrange Carrizo & $19,31 \mathrm{~b}$ \\
Hybride H1 & $39,81 \mathrm{a}$ \\
Hybride H2 & $24,81 \mathrm{~b}$
\end{tabular}

Les moyennes de la même colonne affectées par la même lettre ne diffèrent pas significativement au seuil de $5 \%$ (test de Duncan).

[2] Tozlu I., Moore G.A., Guy C.L., Effect of increasing $\mathrm{NaCl}$ concentration on stem elongation, dry mass production, and macro- and micro-nutrient accumulatioin in Poncirus trifoliata, Aust. J. Plant Physiol. 27 (1) (2000) 35-42.

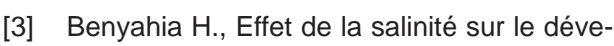
loppement des maladies à Phytophthora des agrumes au Maroc, Univ. Cadi Ayyad, Fac. Sci. Semlalia, Thèse, Marrakech, Maroc, 1998, $170 \mathrm{p}$.

[4] El Guilli M., Benyahia H., Jrifi A., Besri M., Effet de la salinité de l'eau d'irrigation sur la sévérité de la gommose du tronc d'agrumes due à Phytophthora citrophthora, Fruits 55 (3) (2000) 181-186.

[5] Ream C.L., Furr J.R., Salt tolerance of some citrus species, relatives, and hybrids tested as rootstoks, J. Am. Soc. Hortic. Sci. 101 (3) (1976) 265-267.

[6] Walker R.R., Sodium exclusion and potassium-sodium selectivity in salt-treated trifoliate orange (Poncirus trifoliata) and Cleopatra mandarin (Citrus reticulata) plants, Aust. J. Plant Physiol. 13 (2) (1986) 293-303.

[7] Ait Haddou M.M., Rochdi A., Bousrhal. A., Benyahia H., Benazzouz A., Étude de la tolérance de trois porte-greffes d'agrumes vis-à-vis des ions $\mathrm{Cl}^{-}$et $\mathrm{Na}^{+}$, in: Int. Soc. Citric. (ISC), IXe Congr. Orlando, Floride, 3-7 décembre, Orlando, Florida, USA, 2000.

[8] Fernández-Ballester G., Martínez V., Ruiz D., Cerdá A., Changes in inorganic and organic solutes in citrus growing under saline stresses, J. Plant Nutr. 21 (12) (1998) 2497-2514.

[9] Cheeseman J. M., Mechanisms of salinity tolerance in plants, Plant Physiol. 87 (3) (1988) 547-550. 
[10] Vaissel Y., Biology of halophytes, Acad. Press, New-York, USA, 1972, 492 p.

[11] Hoogland D.R., Arnon D.I., The water culture method for growing plants without soil, Calif. Agr. Exp. Stn. Circ., 347, 1954, 32 p.

[12] Zekri M., Salinity and calcium effects on emergence, growth and mineral composition of seedlings of eight citrus rootstoks, J. Hortic. Sci. 68 (1) (1993) 53-62.

[13] Walker R.R., Blackmore D.H., Sunquing D., Carbon dioxide assimilation and foliar ion concentration in leaves of lemon (Citrus limon L.) trees irrigated with $\mathrm{NaCl}$ or $\mathrm{Na}_{2} \mathrm{SO}_{4}$, Aust. J. Plant Physiol. 20 (2) (1993) 173-185.

[14] Troll W., Lindsley J., A photometric method for the determination of proline, J. Biol. Chem. 215 (1955) 655-661.

[15] Lerner H.R., Amzallag G.N., Friedman Y. Goloubinoff P., The response of plants to salinity: from turgor adjustments to genome modification, Israel J. Plant Sci. 42 (4) (1994) 285-300.

[16] Nieves M., Cerdá A., Botella M., Salt tolerance of two lemon scions measured by leaf chloride and sodium accumulation, J. Plant Nutr. 14 (6) (1991) 623-636.

[17] Hubac C., Guerrier D., Study of the amino-acid composition of two Carex sp.: Carex stenophylla Wahl. $f$ pachystylis (J. Gay) Asch. et Graebn., very resistant to drought, and Carex setifolia Godron non Kunze, low resistance. Effect of application of proline, Oecolog. Plantar. 7 (2) (1972) 147-165.

[18] Hasson E., Poljakoff-Mayber A., Changes in osmolarity and solute content of pea plants exposed to salinity and abscisic acid, Aust. J. Plant Physiol. 10 (6) (1983) 573-583.

[19] Townsend A.M., Response of selected tree species to sodium chloride, J. Am. Soc. Hortic. Sci. 105 (6) (1980) 878-883.

\section{Efecto del estrés salino en la acumulación de prolina y azúcares solubles en hojas de tres patrones de cítricos.}

Resumen - Introducción. La salinidad del suelo y del agua de riego, agravada por la escasez de las precipitaciones, es una amenaza permanente para el cultivo de los cítricos en Marruecos. La elección de un patrón tolerante a este estrés abiótico sería un medio eficaz para paliar este problema. Este experimento permitió evaluar, frente al estrés salino, tres patrones recientemente introducidos en Marruecos. Material y métodos. Se evaluaron plantas de citrange Carrizo y de dos híbridos H1 y H2 procedentes del cruce Poncirus trifoliata x Citrus sunki Hort. ex Tan. en función de su tolerancia a la salinidad. Los plantones de 18 meses de edad de regaron con una solución nutritiva a la que se añadió $(25,35$ y 70$) \mathrm{mM}$ de $\mathrm{NaCl}$ durante uno o dos meses mientras que las plantas testigo recibían la solución nutritiva sin $\mathrm{NaCl}$. Tras tratamiento, se midió en las hojas de los patrones tratados el contenido de prolina y azúcares solubles. Resultados. La acumulación de prolina fue independiente de la presencia de $\mathrm{NaCl}$ en la solución de riego pero varió en función del patrón. En cambio, $\mathrm{NaCl}$ estimuló mucho la acumulación de azúcares solubles en los tres patrones y esta acumulación fue muy importante en el patrón H1. Discusión y conclusiones. Estos resultados coinciden en parte con algunos estudios ya publicados. En nuestras condiciones experimentales, el efecto de las concentraciones salinas utilizadas pudo ser compensado con la acumulación de azúcares solubles en las hojas, especialmente en las del patrón H1. Al haber mostrado éste menos toxicidad foliar que los dos otros patrones, la acumulación de azúcares solubles en la hoja de cítricos podría ser un índice de tolerancia a la salinidad.

Marruecos / Citrus / portainjertos / tolerancia a la sal / extractos de hoja / azucares / prolina

To access this journal online: www.edpsciences.org 